\title{
Bullying unter Geschwistern
}

\author{
Eine Übersicht
}

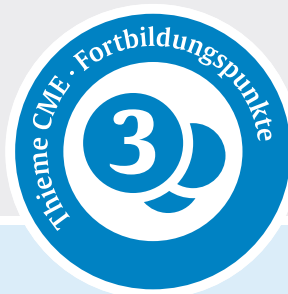

Andreas Witt, Jörg M. Fegert

Klinik für Kinder- und Jugendpsychiatrie und Psychotherapie, Universitätsklinikum Ulm

\section{ZUSAMMENFASSUNG}

Gegenstand und Ziel Gewalt und Bullying unter Geschwistern ist bislang ein wenig beachtetes Thema. Im vorliegenden Beitrag soll ein Überblick über aktuelle Erkenntnisse zum Thema Bullying unter Geschwistern gegeben werden.

Methoden Die vorliegende Arbeit ist eine Übersichtsarbeit, in der aktuelle Literatur zum Thema Bullying unter Geschwistern zusammengefasst wird.
Ergebnisse Studien zeigen, dass Bullying unter Geschwistern ein häufiges Phänomen darstellt. Die Folgen für die Betroffenen sind vergleichbar mit den Folgen von Bullying durch Gleichaltrige. Bislang bestehen kaum evidenzbasierte Interventions- und Präventionsprogramme.

Schlussfolgerung Bullying unter Geschwistern in ein häufiges Phänomen und stellt einen Risikofaktor für die Entwicklung von Kindern und Jugendlichen dar. Die Entwicklung von evidenzbasierten Interventionen ist notwendig.

Klinische Relevanz Aufgrund der Häufigkeit und der Folgen sollte Bullying unter Geschwistern in der Anamneseerhebung beachtet werden.

\section{Die Bedeutung von Geschwister- beziehungen}

Geschwisterbeziehungen stellen neben Beziehungen zu Gleichaltrigen bedeutsame Kontexte für die individuelle Entwicklung von Kindern dar. Sie sind die überdauerndste Form von Beziehungen über die Lebenszeit hinweg [1, 2]. So verbringen Kinder in der mittleren Kindheit mehr Zeit mit ihren Geschwistern als mit ihren Eltern [3]. Zahlen des Statistischen Bundesamtes zeigen, dass in Deutschland die meisten minderjährigen Kinder mit mindestens einem minder- oder volljährigen Geschwisterkind gemeinsam in einem Haushalt leben [4].

Geschwisterbeziehungen können dabei durch Liebe und Zuneigung, aber auch durch Gewalt und Missgunst geprägt sein. Sie haben damit substanzielle und weitreichende direkte und indirekte Einflüsse auf die Entwicklung von Kindern [5]. Positive Geschwisterbeziehungen können dabei eine Vielzahl positiver Einflüsse auf die Entwicklung haben und zum Wohlbefinden beitragen [6]. Doch sind Geschwisterbeziehungen nicht immer harmonisch und unterstützend. Konkurrenz zwischen Geschwistern und gelegentliche Konflikte werden häufig als normal betrachtet. Das Thema Rivalität unter Geschwistern ist ein in der Gesellschaft schon lange präsentes Phänomen, wie etwa die Geschichte um Kain und Abel belegt. Eine substanzielle Zahl von Kindern und Jugendlichen gibt jedoch an wöchentlich Opfer von Bullying durch Geschwister zu werden [6]. Angelehnt an die Definition von Bullying nach Olweus [7] definieren Wolke und Kollegen Bullying unter Geschwistern als Form von Aggression zwischen Geschwistern, die indirekte oder direkte und persistierende Ver- haltensweisen sowie ein Machtungleichgewicht beinhalten [6]. Aggressive Verhaltensweisen können physischer (schlagen), verbaler (Beschimpfungen) oder relationaler (Gerüchte verbreiten) Natur sein. Damit werden einzelne aggressive Verhaltensweisen ausgeschlossen, die eher als Geschwisterrivalität gesehen werden können und damit eine weniger schwere Form darstellen.

\section{Häufigkeit von Bullying unter Geschwistern}

Insgesamt bestehen nur wenige Studien, die Bullying unter Geschwistern untersuchen [6]. Aufgrund einer fehlenden einheitlichen Erfassung von Bullying unter Geschwistern mit unterschiedlichen Messinstrumenten und verschiedenen Cut-Offs ist keine eindeutige Aussage über die Häufigkeit des Phänomens zu treffen. Unbestritten ist jedoch, dass Bullying unter Geschwistern ein häufiges Phänomen darstellt und viele Kinder und Jugendliche davon betroffen sind. Auf Basis der Ergebnisse unterschiedlicher Studien aus den USA, Israel Uk und Italien schließen Wolke und Kollegen, dass Bullying unter Geschwistern eine häufige Form von Misshandlung darstellt [6]. Werden alle von Formen von Bullying unter Geschwistern mit einbezogen, zeigen sich in einer systematischen Literaturübersicht von Wolke und Kollegen Prävalenzraten von 15\% bis $50 \%$ dafür Opfer von Bullying durch Geschwister zu werden. Die Prävalenzraten dafür Bullying gegen Geschwister auszuüben liegen zwischen $10 \%$ und $40 \%$ [6]. Damit liegen diese Zahlen über den Raten, wie sie üblicherweise für Bullying unter Gleichaltrigen gefunden werden [8]. Neben der höheren Frequenz von Bullying unter Geschwistern im Vergleich 
Bullying unter Gleichaltrigen [9, 10], scheint ein Merkmal von Bullying unter Geschwistern die hohe Zahl derer zu sein, die zugleich Opfer und Täter sind [6, 10]. Wolke und Kollegen sehen dies als Ausdruck einer weniger statischen Machtdynamik in Familien [6].

In einer bevölkerungsrepräsentativen Studie aus Deutschland mit 2516 Teilnehmern gaben $73 \%$ an in der Kindheit und Jugend mit einem Geschwister, Halbgeschwister oder Stiefgeschwister zusammengelebt zu haben. Von diesen gaben 12,8\% mindestens einmal pro Monat von ihren Geschwistern absichtlich verletzt oder gemobbt worden zu sein. Selbst andere Geschwister mindestens einmal pro Monat absichtlich verletzt oder gemobbt zu haben, gaben 11,8\% der Befragten an, die mit Geschwistern zusammengelebt hatten. Auch in dieser Untersuchung zeigt sich die hohe Überschneidung von Tätern und Opfern von Bullying. Insgesamt gaben 9,9\% an, mindestens einmal pro Monat Bullying erlebt und selbst ausgeführt zu haben [11].

\section{Massive Formen von Bullying unter Geschwistern}

Während klar ist, dass Geschwister untereinander streiten und Konflikte austragen, kann es unter Geschwistern aber auch zu massiven Ausbrüchen von emotionaler und körperlicher Gewalt kommen. So berichten vier von fünf Familien, dass im letzten Jahr irgendeine Form von Gewalt zwischen den Geschwistern auftrat [12]. Bei einer Vielzahl dieser Familien, fielen diese Formen relativ gering aus und beinhalteten Handlungen, wie Schubsen, Schlagen oder das Werfen von Gegenständen. Ein großer Anteil der Familien (53\%) berichtete jedoch auch von Gewalttaten mit einem hohen Schädigungspotential, wie Treten, Beißen, Schlagen mit Objekten und Schlägereien. Darüber hinaus wurde sogar der Gebrauch von Waffen, wie Messer oder Schusswaffen beschrieben [12]. Während solche Taten im Alltag fast immer zur Anzeige gebracht werden, folgen diesen Taten im familiären Umfeld selten Konsequenzen, sondern bleiben häufig hinter verschlossenen Türen [10, 12].

\section{Folgen von Bullying unter Geschwistern}

Bullying unter Geschwistern kann ähnliche Folgen, wie das Bullying unter Gleichaltrigen auslösen. Manche Autoren beschreiben darüber hinaus, dass Bullying unter Geschwistern im Vergleich zu Bullying unter Gleichaltrigen schwerwiegendere Folgen haben kann. Ein Argument dafür ist, dass Bullying unter Geschwistern auch das Risiko für Bullying durch Gleichaltrige erhöht. Manche Kinder haben somit keine Möglichkeit diesen negativen Einflüssen zu entfliehen und werden zu Hause und auch in der Schule Opfer von Bullying. Die Effekte scheinen kumulativ zu wirken. Kinder, die Bullying durch Geschwister und Gleichaltrige erleben, weisen das größte Risiko für die Entwicklung von Störungen auf [6]. Bullying unter Geschwistern wurde als Risikofaktor für emotionale Probleme, wie Depression in der Adoleszenz identifiziert [6]. Eine prospektive Studie zeigt, dass Bullying unter Geschwistern das Risiko für de- pressive Symptome und Selbstverletzung um das doppelte erhöht [13]. Dieser Effekt bleibt auch bestehen, wenn für verschiedene Faktoren kontrolliert wird.

Neueste Forschungsergebnisse zeigen zudem einen deutlichen Zusammenhang mit psychotischen Störungen [14]. So haben Personen, die als Kinder von Geschwistern gemobbt wurden ein um das Dreifache erhöhte Risiko eine psychotische Störung zu entwickeln. Dabei zeigt sich auch, dass das Risiko für eine psychotische Störung steigt, je häufiger die jungen Erwachsenen als Kinder in Bullying, als Opfer, Täter oder in beiden Rollen verwickelt waren. Das höchste Risiko wurde für diejenigen Kinder beobachtet, die sowohl Täter als auch Opfer waren.

\section{Faktoren für Bullying unter Geschwistern}

Das Zuhause ist der primäre Ort, an dem Geschwister untereinander interagieren. Somit spielen Haushaltsfaktoren eine wichtige Rolle für das Auftreten von Gewalt unter Geschwistern. Erziehungsqualität und das Erziehungsverhalten der Eltern sind dabei die intrafamiliären Faktoren, die am stärksten mit Bullying zwischen Geschwistern assoziiert sind [6]. Insbesondere Kindesmisshandlung wurde als Risikofaktor für Bullying unter Geschwistern identifiziert $[1,13,15,16]$. Auch fehlende elterliche Wärme und Beaufsichtigung sind mit Bullying unter Geschwistern assoziiert [17]. Elterliche Ungleichbehandlung und Bevorzugung von Geschwistern spielen darüber hinaus eine Rolle. Ferring und Kollegen zeigten negative Folgen für die benachteiligten Geschwister sowie für die Geschwister- und Eltern-Kind-Beziehung [18]. Die psychischen Folgen bei den Betroffenen können von Depressionen über Rückzug und psychosomatischen Beschwerden bis hin zu aggressivem und antisozialem Verhalten reichen. Und besonders schwer sind diejenigen Kinder betroffen, die nicht nur von Mutter oder Vater, sondern von beiden Elternteilen benachteiligt und zurückgesetzt wurden [18]. Umgekehrt zeigt sich aber auch, dass gelegentliche Konflikte zwischen Geschwistern und deren konstruktive Lösung auch positive Effekte haben kann [6].

Darüber hinaus zeigt sich, dass Bullying unter Geschwistern häufiger in Haushalten mit männlichen Geschwistern und Familien, in denen die Geschwister sehr nah nacheinander geboren worden sind, ist [1, 19]. Jüngere Geschwister scheinen häufiger Bullying zu erleben. Insgesamt zeigen Studien zudem, dass mit der Anzahl der Geschwister das Risiko für Bullying unter Geschwistern steigt $[13,19]$. Alleinerziehende Eltern und Stiefeltern scheinen das Risiko für Bullying unter Geschwistern nicht zu erhöhen [6]. Angesichts der steigenden Bedeutung von Patchworkfamilien und dass davon ausgegangen werden kann, dass eine Wiederholung von Mustern stattfindet, sollte zukünftig ein besonderer Fokus auf diesem Thema liegen. 


\section{Bisherige Präventions- und Beratungs- ansätze}

Aufgrund der Tatsache, dass Geschwisterrivalität häufig als normal angesehen wird und allgemein die Überzeugung besteht, dass Aggression und Bullying unter Geschwistern normale Übergangsphänomene darstellen, wurde diesem Thema wenig Aufmerksamkeit geschenkt [6, 20]. Damit wurde auch das Thema Interventionen lange vernachlässigt. Somit bestehen keine spezifischen Behandlungs- oder Präventionsprogramme, die gezielt auf Bullying unter Geschwistern ausgerichtet sind. Jedoch wurden Programme zur Verbesserung der Beziehung unter Geschwistern entwickelt [21-24]. Diese beinhalten Module zur Stärkung sozio-emotionaler Kompetenzen, zur Emotionsregulation, interpersoneller Fähigkeiten und Elternberatung zum Umgang mit Konflikten unter Geschwistern. Insgesamt berichten alle Programme positive Effekte auf die Eltern, Geschwister und Geschwisterbeziehungen [6]. Die Programme nutzen eine Vielzahl unterschiedlicher Methoden, wie Verstärkung positiver Kommunikation, Modelllernen und Videos. Die Eltern werden häufig als Kotherapeuten eingesetzt [6]. Gerade unter dem Aspekt, dass das elterliche Verhalten stark mit Bullying unter Geschwistern assoziiert ist, stellen effektive Mediationsstrategien bei Eltern vielversprechende Ansätze dar, die bisher jedoch nur wenig untersucht wurden [6]. Als besonders vielversprechend sehen Wolke und Kollegen [6] ein Programm zur Verbesserung von Geschwisterbeziehungen, das bei Kindern in Pflegefamilien eingesetzt wird [25]. Dieses Programm integriert Module zur Emotionsregulation, ein soziales Kompetenztraining, Ansätze zum Familiensystem und Meditationsstrategien für die Eltern zum besseren Umgang mit Geschwisterkonflikten. In Anbetracht der Häufigkeit des Problems, sollten niederschwellige Angebote über moderne Medien etabliert werden, die möglichst viele Familien erreichen.

\section{Interventionen}

Interventionen müssen zu Hause starten. Dort muss systematisch exploriert werden, welche Faktoren das Bullying begünstigen. In Anbetracht der Folgen für die Betroffenen sind Interventionen dringend notwendig um Bullying unter Geschwistern zu verhindern oder zu reduzieren [6].

\section{FAZIT FÜR DIE PRAXIS}

Bullying unter Geschwistern ist ein häufiges Phänomen und kann für Betroffene lebenslange Folgen haben. Aufgrund dessen sollte Bullying unter Geschwistern Berücksichtigung in der Anamneseerhebung finden. Evidenzbasierte Interventionen und Präventionsprogramme sind notwendig, um dem Problem wirksam vorzubeugen und zu begegnen.

\section{Korrespondenzadresse}

\section{Andreas Witt}

Klinik für Kinder- und Jugendpsychiatrie und Psychotherapie, Universitätsklinikum Ulm

Steinhövelstraße 5, 89075 Ulm

Tel. 0731/50061639

andreas.witt@uniklinik-ulm.de

Literatur

[1] Eriksen S, Jensen V. A Push or a Punch: Distinguishing the Severity of Sibling Violence. J Interpers Violence 2018; 24(1): 183-208.

[2] Finkelhor D, Turner H, Ormrod R. Kid's stuff: The nature and impact of peer and sibling violence on younger and older children. Child Abuse Negl 2006; 30(12): 1401-1421.

[3] Brody G.H. Sibling Relationship Quality: Its Causes and Consequences. Annu Rev Psychol 2018; 49(1): 1-24.

[4] Statistisches Bundesamt (Destatis). Datenreport 2016: Ein Sozialbericht für die Bundesrepublik Deutschland 2016.

[5] Brody GH. Siblings' Direct and Indirect Contributions to Child Development. Curr Dir Psychol Sci 2004 2018; 13(3): 124-126.

[6] Wolke D, Tippett N, Dantchev S. Bullying in the family: sibling bullying. The Lancet Psychiatry 2015; 2(10): 917-929.

[7] Olweus D. School Bullying: Development and Some Important Challenges. Annu Rev Clin Psychol 2018; 9(1): 751-780.

[8] Hymel S, Swearer SM. Four decades of research on school bullying: An introduction. Am Psychol 2015; 70(4): 293.

[9] Tucker C], Finkelhor D, Turner H, Shattuck AM. Sibling and peer victimization in childhood and adolescence. Child Abuse \& Neglect 2014; 38(10): 1599-1606.

[10] Wolke D, Skew A]. Bullying among siblings. Int ] Adolesc Med Health 2012; 24(1): 17-25.

[11] Witt A, Brähler E, Sachser S, Fegert, JM. Sibling bullying in the general population of Germany. Journal of Family Violence; eingereicht.

[12] Straus MA, Gelles RJ, Steinmetz SK. Behind closed doors: Violence in the American family. London: Routledge 2017.

[13] Bowes L, Wolke D, Joinson C, Lereya ST, Lewis G. Sibling bullying and risk of depression, anxiety, and self-harm: a prospective cohort study. Pediatrics 2014; 134(4): e1032-9.

[14] Dantchev S, Zammit S, Wolke D. Sibling bullying in middle childhood and psychotic disorder at 18 years: a prospective cohort study. Psychol Med 2018: 1-8.

[15] Button DM, Gealt R. High risk behaviors among victims of sibling violence. J Fam Violence 2010; 25(2): 131-140.

[16] Witte S, Fegert JM, Walper S. Risk of maltreatment for siblings: factors associated with similar and different childhood experiences in a dyadic sample of adult siblings. Child Abuse Negl 2018; 76: 321-333.

[17] Updegraff KA, Thayer SM, Whiteman SD, Denning DJ, McHale SM. Relational aggression in adolescents' sibling relationships: Links to sibling and parent-adolescent relationship quality. Family Relations 2005; 54(3): 373-385.

[18] Ferring D, Boll T, Filipp S. Elterliche Ungleichbehandlung in Kindheit und Jugend aus der Perspektive des mittleren Erwachsenenalters. Zeitschrift für Entwicklungspsychologie und Pädagogische Psychologie 2003. 
[19] Tucker C], Finkelhor D, Shattuck AM, Turner H. Prevalence and correlates of sibling victimization types. Child abuse \& neglect JID 2013; 37: 213-23.

[20] Krienert JL, Walsh JA. My brother's keeper: A contemporary examination of reported sibling violence using national level data, 2000-2005. J Fam Violence 2011; 26(5): 331-342.

[21] Feinberg ME, Solmeyer AR, Hostetler ML, Sakuma K, Jones D, McHale SM. Siblings are special: Initial test of a new approach for preventing youth behavior problems. Journal of Adolescent Health 2013; 53(2): 166-173.

[22] Kennedy DE, Kramer L. Improving emotion regulation and sibling relationship quality: The more fun with sisters and brothers program. Family Relations 2008; 57(5): 567-578.

[23] Pickering JA, Sanders MR. The protocol for a randomised controlled trial (RCT) of a brief intervention for parents of children experiencing sibling conflict. Clin Psychol 2018; 20(2): 86-93.
[24] Smith J, Ross H. Training parents to mediate sibling disputes affects children's negotiation and conflict understanding. Child Dev 2007; 78(3): 790-805.

[25] Linares LO, Jimenez J, Nesci C, Pearson E, Beller S, Edwards N, et al. Reducing sibling conflict in maltreated children placed in foster homes. Prevention Science 2015; 16(2): 211-221.

Bibliografie

DOI https://doi.org/10.1055/a-0815-6562

Nervenheilkunde 2019; 38: 17-20

(c) Georg Thieme Verlag KG Stuttgart · New York ISSN 0722-1541 


\section{Punkte sammeln auf CME.thieme.de}

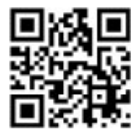

Diese Fortbildungseinheit ist in der Regel 12 Monate online für die Teilnahme verfügbar. Den genauen Einsendeschluss finden Sie unter https://eref.thieme.de/CXCDJWZ.

Sollten Sie Fragen zur Online-Teilnahme haben, finden Sie unter https://cme.thieme.de/hilfe eine ausführliche Anleitung. Wir wünschen viel Erfolg beim Beantworten der Fragen!

Unter https://eref.thieme.de/CXCDJWZ oder über den QR-Code kommen Sie direkt zur Startseite des Wissenstests.

VNR 2760512019156645317

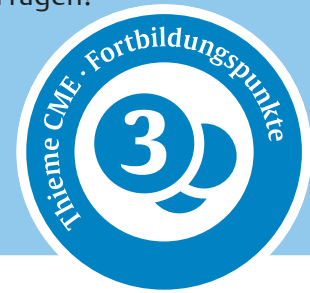

\section{Frage 1}

Welche Aussage ist falsch?

A In der mittleren Kindheit verbringen Kinder mehr Zeit mit ihren Geschwistern als mit ihren Eltern.

B Im Säuglingsalter sind Geschwisterbeziehungen besonders bedeutsam.

C Geschwisterbeziehungen sind bedeutsame Entwicklungskontexte.

D Geschwisterbeziehungen sind die überdauerndste Form von Beziehungen über die Lebenszeit hinweg.

E In Deutschland leben die meisten minderjährigen Kinder mit mindestens einem Geschwisterkind in einem Haushalt.

\section{Frage 2}

Was ist kein Aspekt der Definition von Bullying unter Geschwistern nach Wolke et al. 2015 [6]?

A Bullying beinhaltet indirekte, direkte und persistierende Verhaltensweisen.

B Bullying beinhaltet ein Machtungleichgewicht.

C Bullying kann physische, verbale oder relationale Verhaltensweisen beinhalten.

D Einzelne aggressive Verhaltensweisen sind nicht mit eingeschlossen.

E Bullying ist gleichzusetzen mit Geschwisterrivalität.

\section{Frage 3}

Welche Aussage zur Häufigkeit von Bullying unter Geschwistern ist falsch?

A Die Häufigkeit von Bullying unter Geschwistern wurde bisher noch wenig untersucht.

B Eindeutige Aussagen zur Häufigkeit sind möglich.

C Prävalenzzahlen, Opfer von Bullying zu werden, liegen zwischen 15 und $50 \%$.

D Die Prävalenz von Bullying gegen Geschwister auszuüben liegt zwischen 10 und $54 \%$

E Die Prävalenzzahlen für Bullying gegen Geschwister ist höher als die Zahlen für Bullying unter Gleichaltrigen.

\section{Frage 4}

Welche Aussage zu massiven Formen von Gewalt unter Geschwistern ist richtig?

A Gewalttaten mit hohem Schädigungspotenzial treten unter Geschwistern nie auf.

B Tritt Gewalt mit dem Gebrauch von Waffen unter Geschwistern auf, werden diese fast immer zur Anzeige gebracht.

C Gewalt zwischen Geschwistern ist häufig und tritt meist in relativ leichten Formen auf.

D Massive Formen von Gewalt unter Geschwistern werden häufig öffentlich diskutiert.

E Auf massive Formen von Gewalt zwischen Geschwistern wird meist direkt mit angemessen Konsequenzen reagiert.

\section{Frage 5}

Welche Aussage ist falsch?

A Geschwisterbeziehungen können positive Effekte auf die Entwicklung haben.

B Geschwisterbeziehungen können zum Wohlbefinden beitragen.

C Das Thema Rivalität unter Geschwistern ist in der Gesellschaft schon lange präsent.

D Eine Geschwisterbeziehung kann selbst gewählt werden.

E Geschwisterbeziehungen können sich über die Lebensspanne hinweg verändern.

\section{Frage 6}

Welche Aussage zu den Folgen von Bullying ist falsch?

A Bullying unter Geschwistern kann ähnliche Folgen für die Betroffenen haben, wie Bullying unter Gleichaltrigen.

B Durch Bullying unter Geschwistern ist auch das Risiko für Bullying unter Gleichaltrigen erhöht.

C Die Effekte von Bullying unter Geschwistern und Bullying unter Gleichaltrigen scheinen kumulativ zu wirken.

D Bullying unter Geschwistern ist ein Risikofaktor für die Entwicklung von Störungen.

E Alle Aussagen sind falsch. 


\section{Punkte sammeln auf CME.thieme.de}

Fortsetzung ...

\section{Frage 7}

Für welche Störung wurden bisher keine hohen Zusammenhänge zu Bullying unter Geschwistern gefunden?
A Depression
B Selbstverletzendes Verhalten
C Emotionale Probleme
D Psychotische Störungen
E Ausscheidungsstörungen

\section{Frage 8}

Kein Einflussfaktor für das Auftreten von Bullying unter Geschwistern ist ...
A die Erziehungsqualität der Eltern.
B das Erziehungsverhalten der Eltern.
C elterliche Wärme.
D fehlende elterliche Beaufsichtigung.
E die Ungleichbehandlung von Geschwistern.

\section{Frage 9}

Welcher Faktor begünstigt Bullying unter Geschwistern?

A Haushalte mit weiblichen Geschwistern.

B Familien, in denen die Geschwister einen geringen Altersabstand haben.

C Eine geringere Anzahl von Geschwistern.

D Gemeinsam erziehende Eltern.

E Schulalter der Kinder.

\section{Frage 10}

Welche Aussagen zu Interventionen ist richtig?

A Dem Thema Bullying unter Geschwistern wird in der Forschung große Aufmerksamkeit geschenkt.

B Es bestehen spezifische Interventionen.

C Es bestehen spezifische Präventionsprogramme.

D Programme zur Verbesserung der Beziehung unter Geschwistern enthalten unter anderem Module zur Emotionsregulation.

E Interventionen sollten sich nur an eine geringe und sehr spezifische Gruppe von Familien wenden. 